\title{
Will there be funds to support essential clinical genetic services?
}

In recent years, there has been an enormous increase in our understanding of the genetic basis of human disease. This has permitted much more accurate diagnoses of many diseases, such as Duchenne muscular dystrophy. At the same time, genes have been identified which confer a much greater risk for developing much more common, and adult-onset disorders, such as breast cancer. Historically, medical geneticists have worked with relatively rare conditions, such as phenylketonuria, galactosemia, and Down syndrome, for which the volume of clinical services has not been a major issue confronting the clinical geneticist.

As this new genetic information has been rapidly accumulating relevant to many patients, recommendations have been made to provide genetic testing to large populations, using sophisticated technology, and requiring complex counseling. This has prompted considerable dialogue about who will provide all this necessary clinical genetic service. The exact role of primary care physicians and other practitioners, specialty physicians (for example an oncologist with regard to cancer testing), and importantly the role of the medical geneticist and the genetic counselor will need to be defined.

The article by Pletcher et al. ${ }^{1}$ in this issue of Genetics in Medicine goes a long way to shed light about where the current geneticists are located, exactly what they are doing, how they allocate their time, and what is their current capacity for additional services. Although the survey(s) were undertaken as a part of the Future of Pediatric Education projects, all persons who are certified by the American Board of Medical Genetics in Clinical Genetics were queried. The results confirm some longheld impressions: the majority of geneticists are nonminority, and they work in medical schools in urban environments in practice sites well known to have very high overheads and that also attract poorly funded complex patients. Ninety percent of the patients seen by the geneticists surveyed are children, which reflects the historical beginnings of clinical genetics in pediatrics. This is changing rapidly as we define useful genetic information concerning common, adult-onset disorders.

Instead of focusing on the issue of who will provide genetic services (which I agree is of great importance), I will address what I think is a central issue for anyone who might provide genetic services, i.e., How can we make available clinical genetic services under our current reimbursement programs, and pay the clinical geneticist or other professional person a reasonable salary? Although geneticists are paid salaries that are among the lowest in U.S. medical schools (where most work), these institutions are currently providing clinical genetic services for much less that the salaries paid to the folks who are providing such services. In the long run, this is not sustainable.

Among the very important issues is the very long time required to evaluate a genetic patient. Interestingly, according to the previous and the current study, the time required to evaluate a new genetic patient has dropped from 7.1 hours in $1987,{ }^{2}$ to the current level of 3.1 hours. ${ }^{1}$ This is an enormous change, and such marked positive change suggests that there may well be opportunity to reduce even the current average of 3.1 hours even further. We must be aggressive in ensuring that clinical geneticists make maximal use of electronic aids (e.g., voice activated dictation and the like) to assist in record keeping, and communication, as well as some of the excellent online information services, such as Gene-Clinics. Even with the greatest efficiency, the time required for genetic consultations will remain very long due to the complexity of genetic disease, the time required to research the many rare genetic diseases, the rapid-fire advances in genetics, and the time required to explain complex genetic information to patients and their families. The time required for initial genetic evaluation will not likely ever be as short as for most other medical conditions. The recent changes in demography in major metropolitan areas have added a new and significant expense, i.e., the requirement for translations into many languages in many of our major clinics, such as Miami. Many patients are in health maintenance organizations (HMOs) or in publicly funded programs, where reimbursement, when calculated per hour of professional time and after deducting institutional expenses, will very likely yield direct salary support nearing the U.S. minimum wage.

We must have a clear plan to ensure the long-term viability of clinical genetics as a leading discipline. It is always helpful when clinical geneticists are a part of a team working with a very successful genetic laboratory to provide services. The expert clinical geneticist can work closely with the laboratorian to discuss the tests required and the results of tests as they emerge. Not only does efficiency come from this type of relationship, it also ensures that the patient has the best information, and at the same time permits the laboratory, which usually is far better funded, to support some of the clinical geneticists salary. This model, which works very well in some locations, is being threatened as HMO providers have contracts with large national laboratories, which are distant and unrelated to the local professional geneticist.

Geneticists benefit by working in teams, with others providing as much help as possible in gathering records and other information, filing reports, and securing expert resources and other information. Many medical geneticists already work 
closely with professional genetic counselors, which adds greatly to the efficiency and effectiveness of the services.

We must accurately define what we are doing, break down genetic evaluations, and develop definitive codes for each service. It has been an important goal for the American College of Medical Genetics to work within the Current Procedural Terminology (CPT) Committee to identify and create codes to define genetic services. The College, through its membership in the American Medical Association House of Delegates now has input into these coding committees. Considerable progress has been made with regard to certain laboratory codes. The College Committees are working hard to describe exactly what each of our clinical services include, for example, codes for pedigree and risk assessment (under way), and for other defined services. Currently, we provide an array of diagnostic and counseling services in one very long session, which is not easily described and encapsulated into a single diagnostic code. Perhaps defining the various aspects of a comprehensive genetic evaluation will result in more detailed CPT descriptions, but it might well result in dividing our sessions into several smaller units rather than one very large one. This is much more like other professional services. Families might also well be able to deal better with several encounters, and education might be more effective.

There is one final and important mechanism that we should pursue to enhance our reimbursement for genetic services. I think it is generally agreed in the broad health care environment that the developments in medical genetics have the potential for changing virtually every aspect of clinical medicine. Many of our leading clinical geneticists are faculty and staff of the largest and most prestigious health care providers in major metropolitan areas, where they are responsible for a significant portion of the health care delivered in these areas. Great op- portunities exist here. The geneticists need to be sure that their local practice groups understand and appreciate the great value of genetic services. When these large health care providers are negotiating with the various HMOs and insurance companies, they can include in their program realistic descriptions and reimbursements for important areas such as clinical genetics. Because these large providers have such great prestige and market power, the insurers are likely to accept their recommendations for an area such a medical genetics, which will not likely affect greatly the insurance costs in an area. If these large providers are able (and they will be able to accomplish this) to negotiate realistic rates for clinical genetics, the carriers around the country will likely follow.

In summary, who and how we will provide clinical genetic services remains an important issue to be solved. It is encouraging that this article ${ }^{1}$ reports that many of the fully trained geneticists could see more patients. However, in order for this clinical specialty to grow and thrive, in a time of unparalleled need for genetics services, it is essential that we are aggressive in devising reimbursement strategies that will ensure that those providing clinical genetic services are properly reimbursed for their very special expertise and hard work.

$$
\begin{array}{r}
\text { R. Rodney Howell, MD } \\
\text { Department of Pediatrics } \\
\text { University of Miami School of Medicine } \\
\text { Miami, Florida }
\end{array}
$$

\section{References}

1. Pletcher BA, Jewett EAB, Cull WL, Brotherton SE, Hoyme HE, Pan RJD, Mulvey HJ. The practice of clinical genetics: a survey of practitioners. Genet Med 4:142-149.

2. Bernhardt BA, Weiner J, Foster EC, Tumpson JE, Pyeritz RE. The economics of clinical genetic services, II: a time analysis of a medical genetic clinic. Am J Hum Genet 1987;41:559-565. 